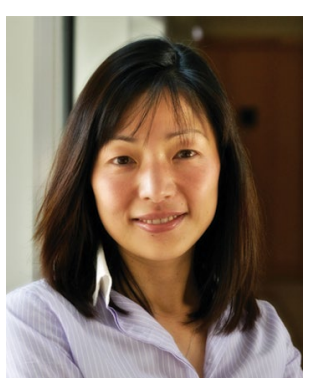

\title{
Antidote to toxic principal investigators
}

There is a pervasive problem in academia of principal investigators who abuse their powers and mistreat their trainees. Here I share suggestions that would help protect the trainees and ultimately reduce the number of toxic supervisors.

A $s$ an advocate of the next generation of scientists, I actively engage with students, postdocs and junior faculty on a daily basis within my institution. In my 20 years of mentoring at the Yale University School of Medicine, I have helped numerous trainees cope with having to deal with toxic principal investigators (PIs). More recently, I have expanded my outreach to trainees globally through social media. In January of this year, I tweeted "When a student sheepishly asks me if she can have a few minutes of my time because she is facing difficulty with her PI, this usually is a sign of significant distress. I know this from experience. If you are in this situation, my DM is open for you. Even if you live far away." This posting received thousands of likes and retweets, and hundreds of PIs poured in to open up their DMs to trainees. I woke up the next morning to find my DM box exploding with messages from students and postdocs suffering from abusive PIs all over the world. These trainees are afraid to reveal their names, institution or anything that can be traced back to the PI, for fear of retribution. Many had reached out to their institutional ombudsperson but had found no clear guidance or solution. The root of the problem is the academic power structure. PIs can hold trainees hostage through letters of recommendation and publications. International trainees are even more vulnerable, because PIs can hold them hostage with their visas. I have seen numerous DMs describing PIs saying "if you don't work harder, I will not be writing a good letter of recommendation for you" or "since you took the weekend off, I am tempted not to renew your visa" (paraphrased). Other PIs simply do not care about their trainees at all.
This paints a global problem in academia_currently there is no clear way to (1) prevent trainees from joining toxic labs, (2) help trainees who are already in such labs, or (3) reform or remove such PIs from academia. Here, I focus on graduate students and recommend antidotes. Solutions for postdoctoral trainees will be discussed elsewhere.

To ensure protection of graduate students from toxic PIs, we can leverage the existing structures of thesis committee and the director of graduate studies (DGS). The DGS should ensure that members of the thesis committee be as diverse as possible, to ensure the inclusion of someone of senior rank who can speak up against the PI, if needed. For students to have regular access to committee members, programs should mandate that committee meetings be held at least once a year per student. This way, PIs cannot prevent students from holding their committee meetings. A standardized committee meeting form should be used so that every student is evaluated by the same criteria. I suggest including an explicit evaluation of the PI's mentorship in such forms. Set aside time for the PI to step outside the room, so the committee can discuss his or her mentorship with the student. Confidentiality should be maintained, and the student should be encouraged to speak of any concerns related to the PI or the lab environment. If a problem is identified, the committee should evaluate the situation through third-party consultation. If the problem is validated, the chair of the department should be informed, and a remediation plan should be formulated and followed through. If necessary, the chair of the department, the DGS and/or the thesis committee members should supply the letter of reference for the student. Finally, we should ban the practice of students' supplying food and drinks for the committee meetings. This poses unnecessary financial and time burden on the students, who are already stressed about the meeting.

How do we prevent harmful behaviors by PIs? The chair of each department should state explicitly the expected faculty conduct in terms of their treatment of trainees in their labs, and discuss the consequences of deviating from good mentoring practice. The promotion of PIs should take into account the mentorship-assessment portion of the thesis committee meeting reports. Mentorship evaluation should be taken into account for tenure decisions and annual salary decisions for all faculty members. On the other hand, exemplary mentors should be duly rewarded with a salary increase and promotion. After all, the goal of academia is the pursuit of knowledge and scholarship_-something that can be fully achieved only through safe and diverse culture.

Akiko Iwasaki (D) 1,2,3

${ }^{1}$ Department of Immunobiology, Yale University School of Medicine, New Haven, CT, USA.

${ }^{2}$ Department of Molecular, Cellular, and Developmental Biology, Yale University, New Haven, CT, USA. ${ }^{3}$ Howard Hughes Medical Institute, Chevy Chase, MD, USA.

e-mail: akiko.iwasaki@yale.edu

Published online: 9 April 2020

https://doi.org/10.1038/s41591-020-0831-6

Competing interests

The author declares no competing interests.

Additional information

Supplementary information is available for this paper at https://doi.org/10.1038/s41591-020-0831-6. 